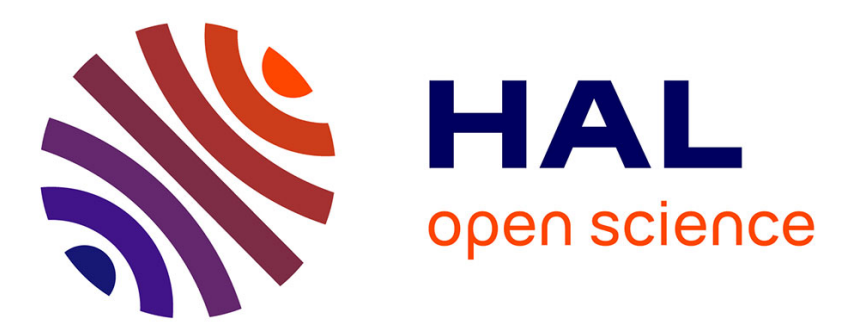

\title{
Visualization and Characterization of Heterogeneous Water Flow in Double-Porosity Media by Means of X-ray Computed Tomography Porous media
}

Z. Peng, Céline Duwig, P. Delmas, Jean-Paul Gaudet, A. Gastelum Strozzi, P. Charrier, H. Denis

\section{To cite this version:}

Z. Peng, Céline Duwig, P. Delmas, Jean-Paul Gaudet, A. Gastelum Strozzi, et al.. Visualization and Characterization of Heterogeneous Water Flow in Double-Porosity Media by Means of X-ray Computed Tomography Porous media. Transport in Porous Media, 2015, pp.art. 0572. 10.1007/s11242-0150572-z . ird-01202110

\author{
HAL Id: ird-01202110 \\ https://hal.ird.fr/ird-01202110
}

Submitted on 18 Sep 2015

HAL is a multi-disciplinary open access archive for the deposit and dissemination of scientific research documents, whether they are published or not. The documents may come from teaching and research institutions in France or abroad, or from public or private research centers.
L'archive ouverte pluridisciplinaire HAL, est destinée au dépôt et à la diffusion de documents scientifiques de niveau recherche, publiés ou non, émanant des établissements d'enseignement et de recherche français ou étrangers, des laboratoires publics ou privés. 


\title{
Visualization and Characterization of Heterogeneous Water Flow in Double-Porosity Media by Means of X-ray Computed Tomography
}

\author{
Z. Peng ${ }^{1}$ - C. Duwig',2 - P. Delmas ${ }^{3}$ - J. P. Gaudet ${ }^{1}$. \\ A. Gastelum Strozzi ${ }^{4}$ - P. Charrier ${ }^{5}$ - H. Denis ${ }^{1}$
}

Received: 25 March 2014 / Accepted: 4 September 2015

C Springer Science+Business Media Dordrecht 2015

\begin{abstract}
Three-dimensional visualization of dynamic water transport process in soil by computed tomography (CT) technique is still limited by its low temporal resolution. In order to monitor dynamically water transport in soil, a compromise has to be found between water flow velocity and CT acquisition time. Furthermore, an efficient image analysis method is necessary. In this work, we followed the water transport in three dimensions by CT imaging across a double-porosity media constituted of two distinct materials, i.e. sand and porous clay spheres. The CT acquisition parameters were adjusted to the water pore velocity so that we succeeded to register the water front displacement per time range of $25 \mathrm{~min}$. We also used the image subtraction method to extract water distribution evolution with time with a space resolution of $6 \times 10^{-3} \mathrm{~cm}$. Both time and space resolution are relatively high compared with other dynamic studies. The water content profiles showed that the clay spheres remained in their dry state during water infiltration, while the water transport only occurred in the sand matrix. These results are consistent with macroscopic experiments. The water front visualized by CT showed a non-symmetrical shape which was related to water transfer in non-equilibrium as shown by column displacement experiments.
\end{abstract}

Keywords 3D visualization - Water transport - Computed tomography · Image analysis . Porous media

\section{Duwig}

celine.duwig@ird.fr

1 IRD/UJF-Grenoble 1, LTHE UMR 5564, BP 53, Grenoble Cedex 9, France

2 LTHE, Bâtiment OSUG-B, Domaine universitaire, BP 53, 38041 Grenoble Cedex 09, France

3 Department of Computer Science, The University of Auckland, PB 92019, Auckland, New Zealand

4 UNAM, CCADET, Ciudad Universitaria, 04510 Mexico Cuidad, Mexico

5 UJF-Grenoble 1, 3SR UMR 5521, BP 53, Grenoble Cedex 9, France 


\section{Introduction}

Understanding water flow transport in porous media is a central issue to natural and engineered soil science. Groundwater and vadose zone flow, contaminant transfer, and energy-related activities such as geothermal energy production depend on the transport properties at pore scale. To study the water transport properties in porous media, traditional methods exist such as water retention (Schjonning et al. 2005; Holtham et al. 2007) and mercury intrusion curves (Lugato et al. 2009) to estimate pore size distribution, or infiltration experiments to estimate hydraulic conductivity (Angulo-Jaramillo et al. 2000). However, these macroscopic techniques have some important drawbacks. These techniques are based on the hypothesis that pores are represented by parallel tubes of different diameters. In the case of water retention and mercury intrusion curves, they cannot provide an estimation of the pore shape as the total porosity is calculated as the sum of accessible pores from the intrusion of the liquid (mercury or water) so that unconnected pores are not detected.

Computed tomography (CT) of X-rays or neutrons provides reliable information at microscale of porous media morphology (Sleutel et al. 2008; Luo et al. 2010; Dal Ferro et al. 2012; Elyeznasni et al. 2012; Munkholm et al. 2012), multi-phase determination of stationary, or transient flow (Hopmans et al. 1992; Wildenschild et al. 2005; Schaap et al. 2008; DiCarlo et al. 2010; Akhtar et al. 2011; Sammartino et al. 2012). According to equipment resolution and sample size, individual grains and fluid interfaces at pore scale can be achieved by CT. Furthermore, many physical and chemical phenomena occur at this scale and determine soil characteristics and transport properties at macroscale.

One particular interest to apply CT is to observe transport phenomena across heterogeneous unsaturated porous media to better understand the impact of porous structure (pore size, connectivity and porosity) on water transport properties. Indeed, the temporal and spatial distribution of water in soil resulting from heterogeneous water flow is affected by the soil structure, i.e. the spatial arrangement of its constituents. When the materials constituting the porous media differ in size and hydraulic properties, the water distribution and flow paths can reveal high complexity. However, the role played by the geometry of solid and voids and the hydraulic properties of the different constituents is still unclear (Vogel and Roth 2003). Since the CT techniques are able to visualize simultaneously the spatial and temporal distribution of solid and liquid phases in 3D (Heindel et al. 2008; Wildenschild and Sheppard 2013), this latter seems to be a good approach to overcome the lack of knowledge. Only a few authors performed spatio-temporal studies of soil behaviour using CT technologies (Schaap et al. 2008; Vasin et al. 2008; Kneafsey et al. 2011; Sammartino et al. 2012). This is both due to the trade-off between acquisition time and image quality and resolution creating a difficult balance between low temporal resolution and acceptable spatial resolution. Consequently, most studies of water transport in transient states were based on 2D radiography (Hopmans et al. 1992; Maruyama et al. 2003; Carminati et al. 2007, 2008; DiCarlo et al. 2010; Esser et al. 2010). Hopmans et al. (1992) studied water infiltration during one-step outflow experiments on repacked homogeneous samples of a sandy soil. Maruyama et al. (2003) recorded the movement of soil water occurring in an Andosol sample using X-ray with a liquid contrast agent. However, the effect of the contrasting agent on the fluid physical and chemical properties and its flow pattern compared to pure water was not discussed. Carminati et al. (2007, 2008) monitored the water exchange in 3D between wet and dry aggregates and simulated the observed water flow and water content distribution with Richards equation using synchrotron-based X-ray tomographic microscopy. 
The 3D measurement of water content distribution in heterogeneous sands during multistep drainage cycles was reported by Schaap et al. (2008) and Vasin et al. (2008). The later used a combination of "fast" 2D neutron transmission tomography to image relatively "slow transient drainage processes" (at a resolution of $0.027 \mathrm{~cm}$ and an acquisition time of $20 \mathrm{~s}$ ) combined with computed tomography to compute a 3D mapping of the studied sample (at the same spatial resolution for an acquisition time of $2 \mathrm{~h}$ ). Kneafsey et al. (2011) proposed an indirect analysis method by observation of density changes of CT signals caused by hydrate formation and water flowing in samples of methane-hydrate bearing sand. Sammartino et al. (2012) used a medical four-slice helical computed tomography to attain fast acquisition of 2D slices at $0.0625 \mathrm{~cm}$ thickness. This method provides new quantitative information on preferential flow processes occurring in an unsaturated undisturbed soil as well as demonstrates the potential for the dynamic imaging of infiltration experiments. However, several obstacles in image quality persist such as low signal-to-noise ratio due to low energy of medical X-ray tomography (to protect the patients) and loss of water detection due to low spatial resolution as typical of commercial medical imaging apparatus.

To our knowledge, a dynamic approach of the 3D visualization of water flow at the microscopic and macroscopic scales (defined as pore scale and sample scale respectively) based on a compromise between spatial and temporal resolution in unsaturated porous media has still not been achieved. Under this context, the goal of this work was to monitor and characterise the 3D water flow path by computed tomography (CT) during dynamic experiments at microscale spatial resolution at regular temporal increments. The CT study in a natural soil reveals many difficulties such as large amount of diverse constituents, low scanned porosity compared with total porosity, and large heterogeneity. As a proof of concept, the natural soil was replaced by a known double-porosity media constituted of spheres made of sintered clay (intra-aggregate pores at nanometre scale) and sand (inter-aggregate pores at micrometre scale). The presence of two different porous media (sand and porous clay spheres) leads to a heterogeneity in structure and impacts hydraulic properties. Consequently, different dynamic processes at local scale between the two pore systems influence the macroscopic behaviour of the media (Lewandowska et al. 2004). Water transport in double-porosity media, by numeric and experimental approaches, has been extensively studied by Lewandowska and colleagues (Lewandowska et al. 2004, 2005a, b, 2008; Szymkiewicz et al. 2008; Szymkiewicz and Lewandowska 2008). They observed that the macroscopic behaviour of the double-porosity media was mainly affected by the degree of heterogeneity of pores (local geometry and conductivity contrast). Nevertheless, their numeric modelling with experimental results was only validated at macroscopic scale and the kinetics at microscopic scale was not checked. To overcome this lack of knowledge, we worked on the visualization and characterization of heterogeneous water flow in a double-porosity media at the microscale interface $\left(6 \times 10^{-3} \mathrm{~cm}\right.$ resolution) by means of X-ray computed tomography from an initial state (dry state) to a saturated state (fully wet) following a infiltration experience. The objective was: (i) to demonstrate the feasibility of CT technique to visualize water flow through heterogeneous porous media in 3D, (ii) to develop an adequate image analysis technique for image acquired at different water content conditions, and (iii) to characterize water flow in the two medium. The developed methodology in this study allows visualizing and characterizing the $3 \mathrm{D}$ water flow at water pore velocity in the range of saturated hydraulic conductivity of fine sand. 


\section{Material and Methods}

\subsection{Sample Preparations}

\subsubsection{Material Characterization}

The double-porosity (DP) media are a physical model composed of sintered clay spheres (biolite: ceramic shell with honeycomb core produced by firing natural clay to temperatures of $1100-1200{ }^{\circ} \mathrm{C}$ in a rotating kiln) and Hostun sand (HN34). Mineralogical composition of Hostun sand is mainly quartz $\left(\mathrm{SiO}_{2}>98 \%\right.$, Desaunay 2011). Its granulometry was characterized by laser diffractometry (Mastersize, Malvern), and its mean grain diameter was $0.0275 \mathrm{~cm}$ with a relatively uniform grain size distribution. The total porosity of sand after packing was around 0.43 (see Table 1).

The clay spheres porosity was determined by mercury porosimetry (AutoPore IV 9500, Micromeritics). Connected porosity was 0.445 with media intra-aggregate pore size $4 \times$ $10^{-6} \mathrm{~cm}$. Solid and bulk densities of sintered clay spheres were also determined by mercury porosimetry with values of 2.38 and $1.32 \mathrm{~g} \mathrm{~cm}^{-3}$ respectively. Average diameter of clay spheres is $0.5 \pm 0.1 \mathrm{~cm}$. The permeability was estimated from mercury intrusion curves through the XDQ code (Xu et al. 1997) with value of $3.3 \times 10^{-12} \mathrm{~cm}^{2}$ which corresponds to a hydraulic conductivity of $3.23 \times 10^{-7} \mathrm{~cm} \mathrm{~s}^{-1}$ for water. This value is six times smaller than that of clay spheres used in Lewandowska et al. (2008) and Tran Ngoc (2008).

\subsubsection{Column}

The double-porosity media (previously dried during $24 \mathrm{~h}$ at $105^{\circ} \mathrm{C}$ ) were filled into a glass column (inner diameter $2.6 \mathrm{~cm}$ and height $10 \mathrm{~cm}, \mathrm{Fig} .1 \mathrm{~b}$ ), by packing the two constituents (sand and clay spheres) in a periodic manner (Fig. 1a) following Lewandowska et al. (2008) and Tran Ngoc (2008). The spheres were carefully arranged by hand in a homogeneous distribution for each layer. Voids between spheres were filled by sand. This process was repeated by placing layer by layer up to fill the whole column (Fig. 1b). Although the spheres and their packing were not completely homogeneous, the average height of the periodic arrangement was $0.87 \mathrm{~cm}$, as controlled by porosity determination from CT scan (twice the distance between porosity maximum on Fig. 1c). Three columns were built up each with an equal weight fraction of sand and clay spheres (Table 2). The total porosity of the DP column was calculated to be around 0.44 whereas the visible porosity at CT scan resolution and pixel averaging was on average 0.18 .

\subsection{Infiltration Experiment and Setup}

The set-up representation is shown in Fig. 1d. The column was vertically connected to a water inlet at the top side and an outlet at the bottom side. At each side, the double-porosity media were maintained by a rigid grid covered with a porous membrane. The boundary condition at inlet, i.e. the water flow rate, was controlled by a peristaltic pump which was connected to a water tank for water supply. The water tank was closed to avoid water evaporation. A constant capillary pressure head of $-60 \mathrm{~cm}$ was imposed at the base of the column, and the leachates returns to the water tank. The weight evolution of water tank was monitored by a Mettler Toledo microbalance connected to a computer. The maximum error of observations was 0.01 $\mathrm{g}$, which corresponds to an accuracy of $\pm 0.002 \mathrm{~cm}$ height of water. The room temperature varied between 23 and $24^{\circ} \mathrm{C}$. 


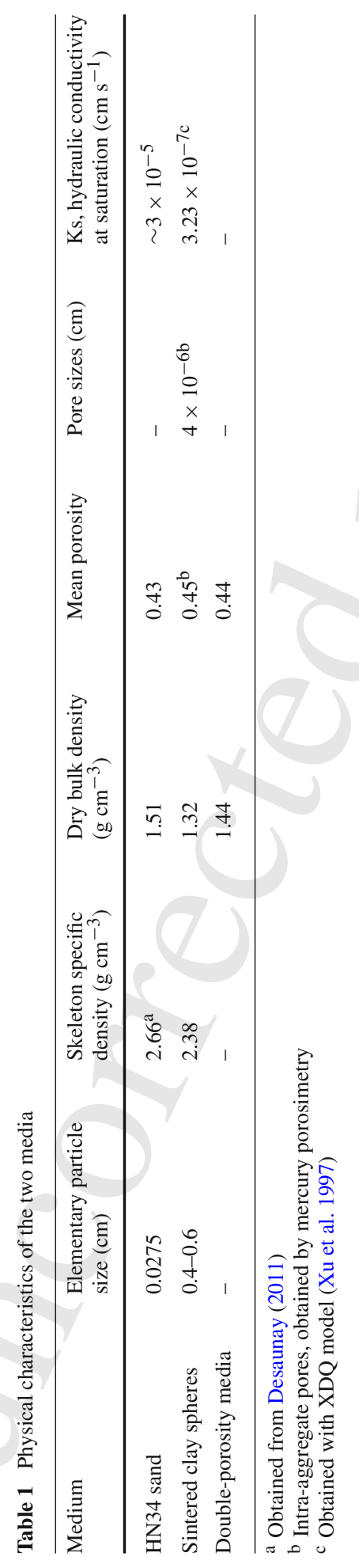




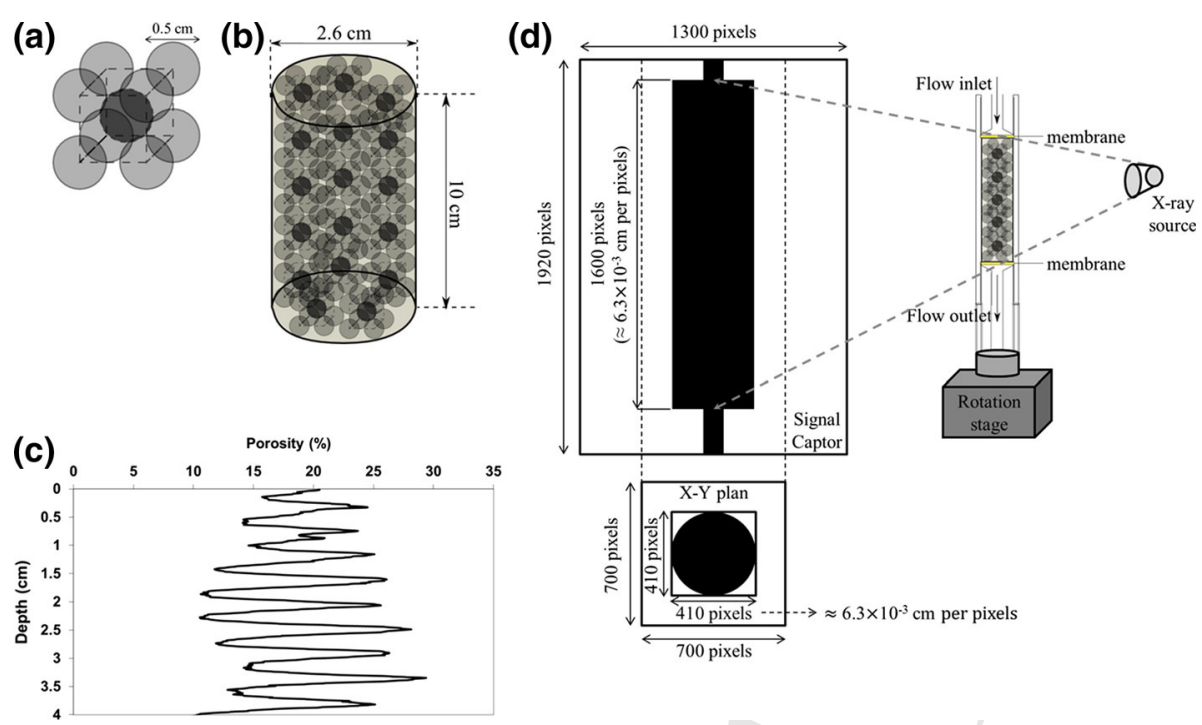

Fig. 1 a Geometric representation of clay spheres arrangement; $\mathbf{b}$ schematic representation of column size; c evolution of porosity as determined by CT scan (for pores $>6.3 \times 10^{-3} \mathrm{~cm}$ ) with depth; $\mathbf{d}$ schematic representation of infiltration setup and its installation in CT measurements

Assuming the weight loss in the water tank is equal to the weight gain in soil column (without taking into account the water volume in capillaries), this latter provides the determination of water content evolution of the soil column as a function of time during transient regime.

Before initiating the dynamic CT imaging experiment, three water flows were tested in soil columns with identical geometry to estimate water infiltration travel time in the soil column. Before each infiltration, the porous media were initially kept air-dry, and the quantity of each media (sand and clay spheres) was separately measured with the microbalance before packing in column. The details of infiltration experience for each column are showed in Table 2.

The dispersivity coefficient of the double-porosity (DP) media was determined during a tracer displacement experiment in column 1, at Darcy water velocity of $6.59 \times 10^{-4} \mathrm{~cm} \mathrm{~s}^{-1}$. When the water flow reached the steady state, a pulse of $\mathrm{KBr}$ solution $\left(670 \mathrm{mg} \mathrm{Br}^{-1}\right)$ of around 1.1 pore volume was injected at the top of the column. The pore volume is defined as the volume of water in the column at steady state. Leachates were collected at the bottom of the column every $5 \mathrm{~min}$, and $\mathrm{Br}$ concentration was analysed with ionic chromatography (Metrohm). Convection-dispersion transport parameters were determined from the $\mathrm{Br}$ breakthrough curve using the CXTFIT 2.1 code (Toride et al. 1999) in inverse mode. A homogeneous HN34 sand column was studied in the same set-up for comparison, with the same Darcy velocity, $\mathrm{KBr}$ solution, and injected pore volume, and the only difference was the leachates collection time (every $10 \mathrm{~min}$ ).

\subsection{CT Scanner Acquisition Parameters}

The infiltration experiment was dynamically imaged using X-ray tomography technologies (TomRX solutions) in the 3SR (Soils, Solids, Structures and Risks) Laboratory, Grenoble, France. The CT scan equipment is calibrated one during the day prior to initiating exper- 


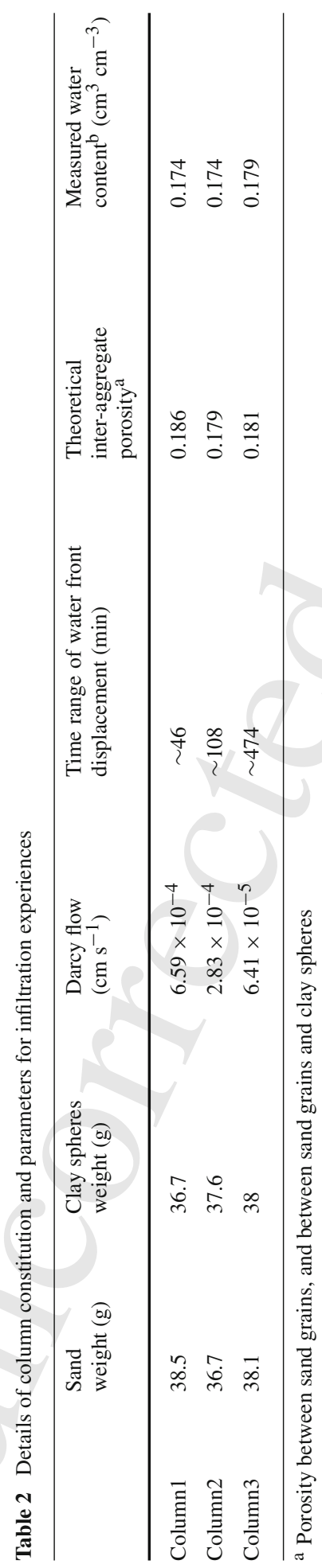


iments. The calibration object consisted of a set of vertically aligned lead spheres which are image with the same CT parameters as our samples, which are later input in the CT reconstruction software. Later the infiltration set-up was installed, and the core column was aligned to the X-ray beams in order to maximize the signal gain onto the captor. The sensor size is of 1300 by 1920 pixels. In order to scan the whole column, the distance from source to objet was adjusted so that the sensor height captured the entire column height, i.e. 1600 pixels for a height of $10 \mathrm{~cm}$, which leads to a spatial resolution of $6.3 \times 10^{-3} \mathrm{~cm}$ in all three dimensions.

Computed tomography generates a three-dimensional image of the inside structure of the studied porous media by acquiring two-dimensional X-ray images $(N)$ taken around a single (vertical) axis of rotation (Herman 2009). To reduce the signal-to-noise ratio, each final $2 \mathrm{D}$ radiographic image is usually computed as the average of $n$ images acquired at the same angular position. Given our experimental constraints, a compromise must be made to minimize the radiographic images acquisition time while maintaining a balanced signal-tonoise ratio and $3 \mathrm{D}$ reconstruction accuracy. The acquisition time $T$ is given by the following equation:

$$
T=N \times n \times \tau
$$

where $N$ is the angular increment onto a defined angular section between 0 and $360^{\circ} ; n$ is the amount of repetition per angular position for the averaging process; $\tau$ is the pause time between two image captures at the same angle.

By performing different tests, the optimal CT acquisition parameters to ensure suitable image quality and acquisition time were found as followed: the value of $\mathrm{N}$ was fixed at 1200 for all the scans onto an angular section of $360^{\circ}$. The value of $n$ which determines the noise level was fixed at 8 . The value of $\tau$ for a significant signal acquisition was adjusted at $0.1 \mathrm{~s}$ (10 images per second) for all the scans.

The photon energy of X-rays (the spot energy) depends on the generator excitation. The relationship between transmission coefficient of each media (gas, liquid, and solid) and photon energy is different: low photon energy leads to high transmission coefficient contrast that allows better distinguishing different media, but low photon transmission due to low photon energy requires long acquisition time. High photon energy leads to high photon transmission and short acquisition time resulting in reduced transmission coefficient contrast (Markowicz 1993). Accordingly, we did set for the whole experiment a tension of $100 \mathrm{kV}$ and a current of $300 \mu \mathrm{A}$ to excite the generator as the best compromise to distinguish between water and sand.

This CT parameter configuration led to a temporal resolution of around 25 min per scan (refer to a full $360^{\circ}$ tomography acquisition). The pore velocity of water was slowed down to achieve a travel time significantly higher than $25 \mathrm{~min}$. In column 3 , we used a low water flow rate $\left(3.4 \times 10^{-5} \mathrm{~cm}^{3} \mathrm{~s}^{-1}\right.$, which corresponds to a Darcy velocity of $6.4 \times 10^{-5} \mathrm{~cm} \mathrm{~s}^{-1}$ or about $2.2 \%$ of saturated hydraulic conductivity of sand) and a theoretical travel time of the water front of $8 \mathrm{~h}$ (see Table 2). The initial CT scan of the column was acquired at dry state. Ten CT scans were performed during the infiltration experiment. Considering the Darcy velocity and the three-dimensional voxel resolution of $6.3 \times 10^{-3} \mathrm{~cm}$ during CT imaging, the vertical water front travelled across each voxel (of thickness $6.3 \times 10^{-3} \mathrm{~cm}$ ) in $94 \mathrm{~s}$. An acquisition time of $25 \mathrm{~min}$ roughly corresponds to an uncertainty on the water front position of 16 voxels. This is equivalent to a resolution at the water front edge close to Sammartino et al. (2012) while achieving a resolution 13 times better anywhere else in the imaged column. The data volume (1920 images slices of 700 by 700 pixels) was reconstructed using the snapCT software which allowed for GPU processing acceleration. Iterative construction and adequate 
filtering reduced reconstruction ring artefacts and beam-hardening effects while preserving the sol core features.

\subsection{Image Processing and Analysis}

The reconstructed soil core data volume was provided as a set of 1920 images encoded in 16 bits greyscale TIFF images with an effective 14-bit intensity dynamic range. This allows for 65,256 shades of grey to encode material density from dark (corresponding to air or vacuum) to white (for material stopping the penetrating $\mathrm{X}$-rays). The images numbered 0 100 and 1700-1919 were first removed as they mostly contained the top and bottom for flow inlet and outlet located above and below the soil sample. The remaining 1600 images were processed using the ImageJ software (Schneider 2012). They were first cropped and cut to remove all non-porous media elements leading to images of dimension 410 by 410 pixels (410 pixels for a width of $2.6 \mathrm{~cm}$, which leads to a spatial resolution of $6.3 \times 10^{-3} \mathrm{~cm}$ ). Three-dimensional median filtering (Serra 1983) was then applied to remove isolated artefacts typically occupying a volume of less than 3 by 3 by 3 voxels while preserving edges of the larger macropores network.

Given the difficulty to use traditional segmentation techniques to separate the macropore system during the infiltration event, a specific technique using subtraction of soil voxels between the dry state and any of the CT volume acquired during the infiltration event is presented in the Sect. 2.5.

\subsection{Wet Pores Network Thresholding}

\subsubsection{Traditional Segmentation Method}

One of the most difficult steps to extract water distribution from the CT image is to threshold the grey level corresponding to water. This latter is not straightforward: on the one hand, the presence of water contributes to a spectral shift showed by the close grey levels between water and dry porous media, due to similar material densities (Fig. 2a). On the other hand, the spectral shift due to water contribution varies as a function of water content contained in the investigated porous media volume (Sammartino et al. 2012). Furthermore, the water distribution in the porous media column is never homogeneous along the vertical axe neither in horizontal plans. Therefore, a fixed threshold value cannot be adapted to extract water across all the slices.

\subsubsection{Subtracted Image Method}

As the traditional segmentation methods could not be applied to our images, we proposed an alternate method based on the subtraction of CT image acquired at dry state from the one acquired at wet state. This method is used currently in petroleum engineering since the late 80's. Withjack (1988) and Akin et al. (2000) determined the porosity by a dual scan at the same location obtained with different fluids saturating the porous medium.

Theoretically, the image obtained from subtraction only contains water signal if the following conditions are satisfied:

(1) The soil sample never moved (above the imaging resolution $-6.3 \times 10^{-3} \mathrm{~cm}$ ) during the infiltration experiment and successive scanning sequences;

(2) The optical configuration of the CT scan never changed during the infiltration experiment; 

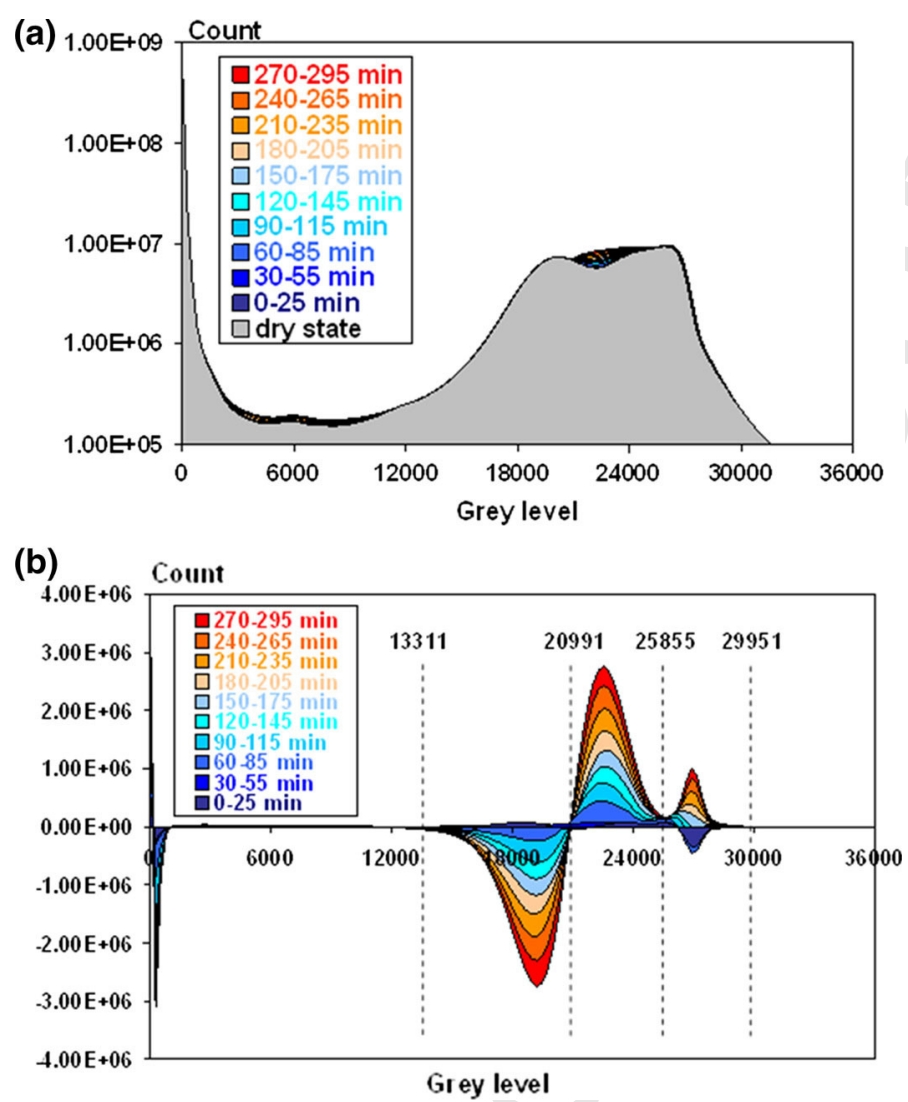

Fig. 2 a Grey-level histograms of the whole column (1600 slices) at different states; b subtraction of dry histogram from histograms at different time range during infiltration experience

(3) The inner soil structure did not change (above the imaging resolution $-6.3 \times 10^{-3} \mathrm{~cm}$ ) during the infiltration experiment and successive scanning sequences.

Both conditions (1) and (3) were verified following the same process: the physical imprints of the column container (condition 1) and of the sand grains in the soil core (condition 3) were compared before the infiltration experiment start (first CT scan) and at the saturated state (last CT scan). The plastic container and the sand grains were isolated out of the soil core in both first and last CT scan data using traditional segmentation methods (e.g. histogram segmentation). The resulting binary images for the first and last CT scan data were subtracted. The resulting difference for the plastic container only displayed isolated pixels likely representing acquisition noise and/or actual displacement of the studied structure below the imaging resolution $\left(6.3 \times 10^{-3} \mathrm{~cm}\right)$. This confirmed condition 1 . The difference image of the sand grains exhibited a set of pixels located at the periphery of some of the sand grains. Overall this did not account for more than a few per cent of the overall sand grains pixels count and it was considered that condition 3 is loosely verified up to the imaging resolution. The condition (2) was checked by following the evolution in time of the grey-level histogram of the glass tube in which the porous media were packed. As the glass tube is completely inert to its inner content, the grey level of the glass tube contour is invariant whatever the 


\section{Slice $n^{\circ} 1000$ (in the middle of the column)}

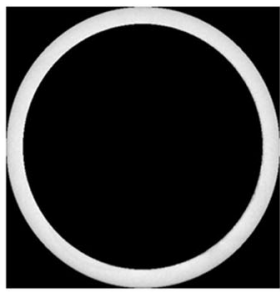

Glass layer of the column

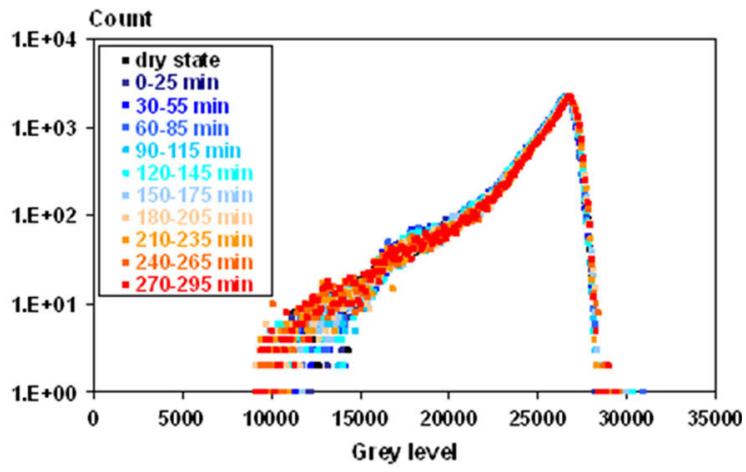

Gray level histograms of the glass layer

state of its inner content. As consequence, the only possibility leading to a variant grey level of the glass tube is the optical configuration change. As the observed grey level of the glass tube was found constant, the condition (2) is satisfied. An example of the slice no. 1000 (the slice situated at the middle of the column) is shown in Fig. 3: the grey-level histograms of this slice displayed the same distribution during the infiltration experience. This validates our hypothesis.

\section{Results and Discussions}

\subsection{Hydrodynamic Parameters of the Double-Porosity Media}

The gravimetrical measurement of water content during infiltration is shown in Fig. 4a. In HN34 sand media, water filled into the inter-aggregate pores until the steady state was achieved with a water content of $0.29 \mathrm{~cm}^{3} \mathrm{~cm}^{-3}$ which is lower than the inter-aggregate porosity of HN34 sand (0.44). This latter can be explained by the Darcy flow applied of $6.59 \times 10^{-4} \mathrm{~cm} \mathrm{~s}^{-1}$ which is lower than the saturated conductivity of HN34 sand and by the imposed lower boundary condition. In the double-porosity (DP) media, two phases were observed with different water infiltration kinetics. During the first infiltration phase, the increasing weight of infiltrated water was rapid and stable; the water content at the end of the first phase seems to be independent of water flow rates. In the second phase, the water infiltration was significantly slowed down. We cannot explain the small peak between the two phases.

By knowing the sand/clay spheres quantities (see Table 2), bulk density and porosity of each media (Table 1), we calculated the inter-aggregate voids volume of the double-porosity column (i.e. not taking into account the intra-aggregates voids inside the clay spheres). We found an inter-aggregate porosity of $0.18 \mathrm{~cm}^{3} \mathrm{~cm}^{-3}$, whereas the water content at the end of the first phase was around $0.17-0.18 \mathrm{~cm}^{3} \mathrm{~cm}^{-3}$ whatever the soil column. We can conclude that for the first phase, the water filled only the inter-aggregate porosity and did not enter the clay spheres. This water content value also corresponds to the average visible porosity as determined by CT. 

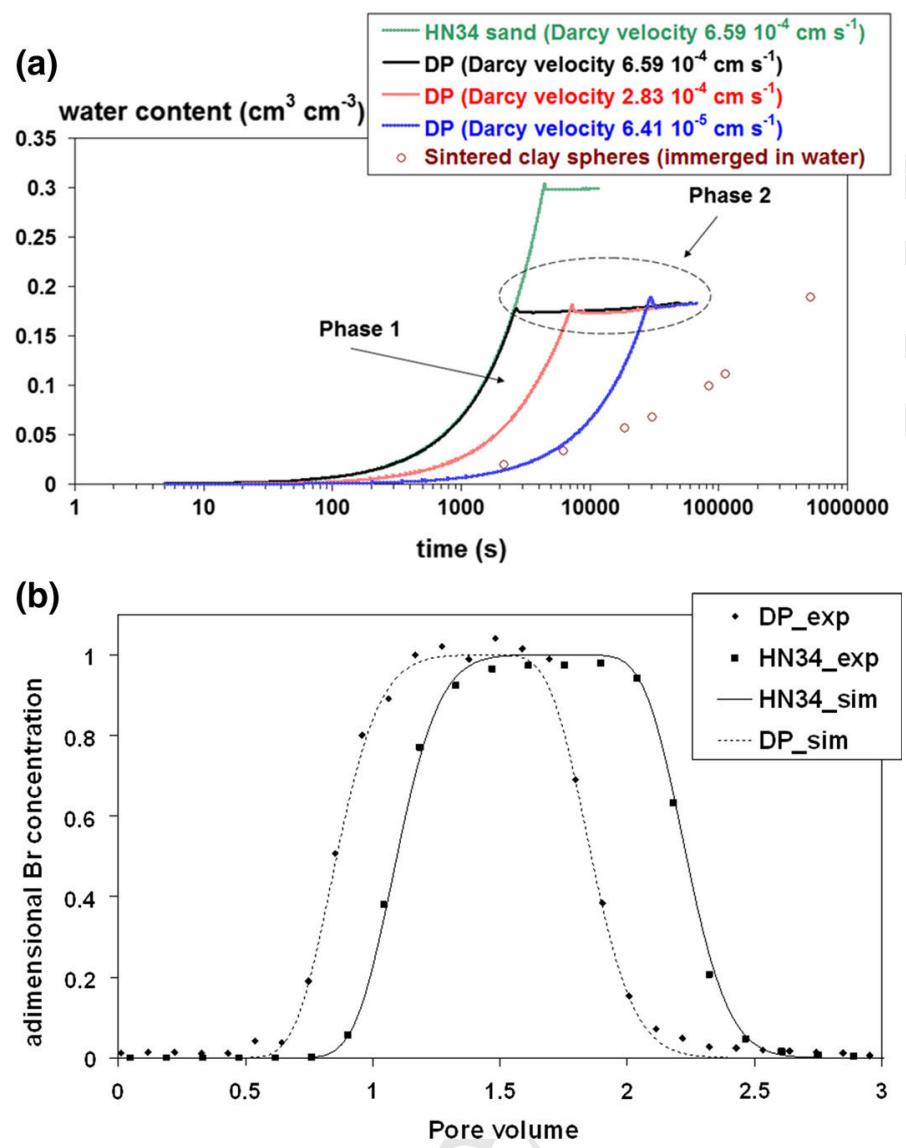

Fig. 4 a Gravimetrical measurement of water content as function of infiltration time for different columns; b experimental (exp) and simulated (sim) breakthrough curves in the double-porosity (DP) and sand (HN34) column, with Darcy velocity of $6.5910^{-4} \mathrm{~cm} \mathrm{~s}^{-1}$

For the second phase, the slow increase in water content in the DP media can be due to filling of water into the clay spheres, at long infiltration time. By comparison, the water content in the HN34 sand media which were obtained in the same set-up did not increase when steady state was achieved. To verify this latter hypothesis, a simple test was performed by immerging 13 clay spheres in water and weighting them at different time intervals. Water content evolution with time of the 13 clay spheres is also shown in Fig. 4a. Even immerged in water, the water infiltration kinetic in clay spheres is slower than the infiltration kinetic in HN34 sand or DP media. This result shows that the increase in water content observed in the second phase in the DP media is certainly due to water infiltration inside clay spheres. One should be noted that this infiltration of water in clay spheres occurred at very large timescale which is out of our CT experience as described later.

To determine the hydrodispersive parameters of the DP media, the breakthrough curve (BTC) of Br was registered after the gravimetrical water content measurement in DP column 1 in which the Darcy flow was $6.59 \times 10^{-4} \mathrm{~cm} \mathrm{~s}^{-1}$. For comparison, another BTC was registered in HN34 sand column with the same Darcy flow. In permanent regime, the water contents were 0.188 and $0.29 \mathrm{~cm}^{3} \mathrm{~cm}^{-3}$ for DP and HN34 sand column respectively. Br transport in 
the HN34 sand column was in equilibrium, with a retardation of 1.1, and a dispersivity of $0.07 \mathrm{~cm}$. As observed on Fig. 4b, Br BTC in the DP media goes out earlier than Br in the sand. Supposing that Br retardation was the same in the sand and in the DP media, the equilibrium convection-dispersion equation could not be fitted to the Br BTC in the DP media. By fitting the non-equilibrium convection-dispersion equation, we found a dispersivity of $0.13 \mathrm{~cm}$, a mobile water content ratio of 0.86 , and solute exchange rate between mobile and immobile regions of $6.6 \times 10^{-12} \mathrm{~s}^{-1}$. For comparison, Tran Ngoc (2008) found a dispersivity value of $0.69 \mathrm{~cm}$ in their DP media under unsaturated conditions $\left(0.313 \mathrm{~cm}^{3} \mathrm{~cm}^{-3}\right)$ to be compared to a dispersivity of $0.044 \mathrm{~cm}$ in the sand at a water content of $0.355 \times \mathrm{cm}^{3} \mathrm{~cm}^{-3}$ (Sato et al. 2003). Although the water content and the Darcy velocity in our experiment were lower, and knowing that Tran Ngoc worked with a finer sand (HN38, with a mean grain diameter of $0.0162 \mathrm{~cm}$ ), we found the same increase in dispersivity in the heterogeneous medium compared to the homogeneous medium (Toride et al. 2003) .

In our experiment, we applied a pulse input and the shape of BTC in the DP media was slightly dissymmetrical with an early breakthrough of $\mathrm{Br}$ compared to one pore volume, while it shows a typical convective/dispersive feature in HN34 sand (Fig. 4b). Bromide transport in the DP media was in physical non-equilibrium, with $14 \%$ of the water content not participating in the transport. The exchange between immobile and mobile water is very slow (rate of $6.6 \times 10^{-12} \mathrm{~s}^{-1}$ ) explaining the nearly symmetrical curve. Tran Ngoc (2008) registered the BTC by applying the water tracer as a step input in homogeneous media (sand) or heterogeneous media (double porosity). They found that the BTC in homogeneous sand was symmetrical, while the BTC in double-porosity media showed no-Fikian characteristics with rapid early breakthrough of the tracer $(\mathrm{NaCl})$ at short times and a drag effect of solute concentration at long time. Furthermore, they also simulated a BTC by assuming that the embedded clay spheres were impermeable; in this case, the breakthrough of tracer concentration was later observed, and the drag effect of solute concentration was reduced even in the presence of clay spheres. They concluded that the early breakthrough and drag effects were due to the presence of porous embedded clay spheres.

Characteristic times were calculated for our DP displacement experiment: resident solute time was $2857 \mathrm{~s}$, whereas the convective time was $2446 \mathrm{~s}$, to be compared to time of exchange between mobile and immobile water zone: $3.9 \times 10^{10} \mathrm{~s}$ and diffusive time: $6.6 \times 10^{10} \mathrm{~s}$. It shows clearly that the main process is the convection during the time of the displacement experiment (total duration of 16,800 s) and that diffusion of water in the clay spheres will happen at longer times. As shown in Table 1, the Ks value of clay spheres used in this work, obtained by XDQ code (Xu et al. 1997), is six times smaller than that of Tran Ngoc (2008). As consequence, the convective transport in the clay spheres is negligible. This led to the nearly symmetrical BTC. According to these macroscopic results, it validates our above results from gravimetric measurements that the water transfer occurred mainly in the pores between sand grains and that the clay spheres were weakly wetted during water transport.

It has to be noted that the Darcy flow applied during infiltration in CT experience was only $6.41 \times 10^{-5} \mathrm{~cm} \mathrm{~s}^{-1}$ which is 10 times smaller than this applied in BTC measurement, in the aim to slow down water front displacement for CT measurements. As a consequence, the water distribution as well as the water transport properties in the column can be different with these two applied flows. In the work of Tran Ngoc (2008), numerous BTC measurements were performed in homogeneous HN38 sand and heterogeneous double-porosity media, at different Darcy flows under unsaturated and saturated conditions. Whatever the homogeneous or heterogeneous media, they showed increasing early breakthrough and drag effects at saturated state compared to unsaturated states. We might thus have a decrease in immobile water content for lower Darcy flow, such as our infiltration experiment in the CT. 


\subsection{Images Analysis and Water Distribution Extraction/Determination}

\subsubsection{Traditional Segmentation Method}

Figure 2a shows the original grey-level histograms of the whole column as a function of infiltration time range. Each histogram is the sum of histograms of 1600 slices forming the column and corresponds to the value registered during the acquisition time range (25 min). In order to clarify the band assignments, the histogram at dry state was subtracted to each histogram at different time range during the infiltration (Fig. 2b). As the histograms mathematically (not spatially) refers to grey-level counts of all the voxels summed at the whole column, the difference of histograms between dry state and infiltration at given time range gives insights into the grey-level band assignments, i.e. the counts of a positive peak is equal to the amount of voxels (with unknown spatial coordination), in which the grey level changed from initial state to a given state according to the experiences. In our case, during infiltration (a wetting process), the two positive peaks (positioned at grey-level bands of 20,991-25,855 and 25,855-29,951) are due to the presence of water which leads to spectral shift towards higher grey level in the range of 20,000-30,000. The adjacent negative peak (grey-level band of 13,311-20,991) corresponds to the loss of initial void (air) that was filled by water during infiltration. As well, the signs of these peaks would be inversed if we performed a drying process (not done). Using the corresponding grey-level band of these peaks, the different phases were not so clearly separated when the images were segmented using traditional techniques (e.g. histogram segmentation using the above grey-level bands limits as lower and upper thresholds). Figure 5 shows the slice no. 1000 at wet state (time range 270-295 $\mathrm{min}$ ), while the whole grey-level distribution is given in Fig. 5a. The grey-level band of 13,311-20,991 (Fig. 5b) which corresponds to pixels representing air also shows a major occupation in the sand, in a clay sphere near the centre of the core. The grey-level band of 20,991-25,855 (Fig. 5c) seems to represent the water in the sand matrix and inside clay spheres. The band of 25,855-29,951 (Fig. 5d) shows the remaining contribution of clay spheres and glass layer.

\subsubsection{Subtracted Image Method}

The subtracted image method is illustrated in Fig. 6 with the slice no. 1000: Fig. 6a corresponds to the dry image and its grey-level values distribution (presented as a histogram); Fig. $6 \mathrm{~b}$ corresponds to wet image of the same CT slice and its respective grey-level values distribution. The resulting subtracted image with positive grey levels (Fig. 6c) showed a distinct separated phase with major water contribution in sand and slight contribution in clay spheres. Given their respective density, the water-filled pixels have higher grey-level values than the air/vacuum filled pixels. Pixels which were filled with air in the reference stack (dry state) and are now filled with water at time t exhibits positive grey-level values on the subtracted image. The materials which were optically and geographically invariant during the infiltration lead to the grey level of 0 . The subtracted image also showed a range of negative grey levels (Fig. 6d). In Sammartino et al. (2012), it was considered that the range of negative grey values may correspond to voxels where the water was initially present but had left at time $t$. However, a negative range of grey level does not have a physical sense in our case, as we used an "artificial" porous media initially at the dry state. The only possibility to explain a negative value in our case is that at a given pixel position, the sand grain moved from several micrometres during water infiltration and was replaced by air or water which results in a negative value while image subtraction. The image of negative grey levels only showed an 
(a)

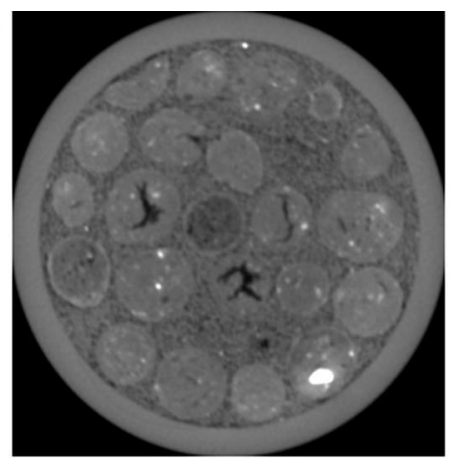

(b)

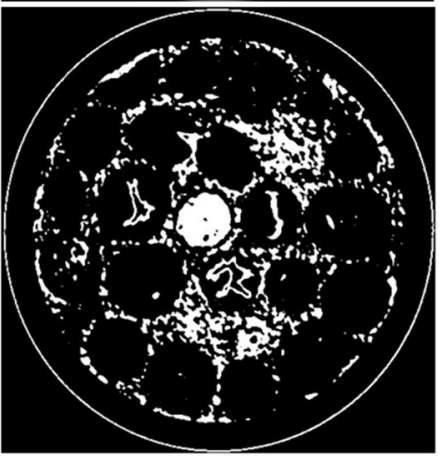

(c)

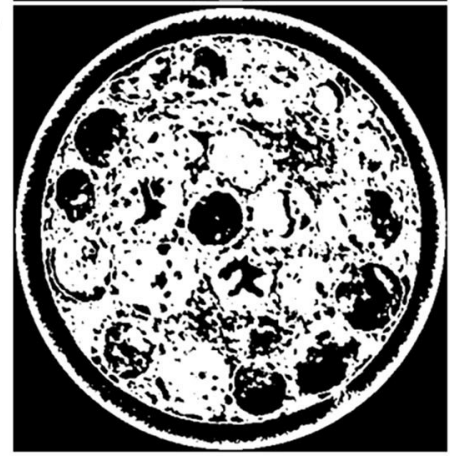

(d)

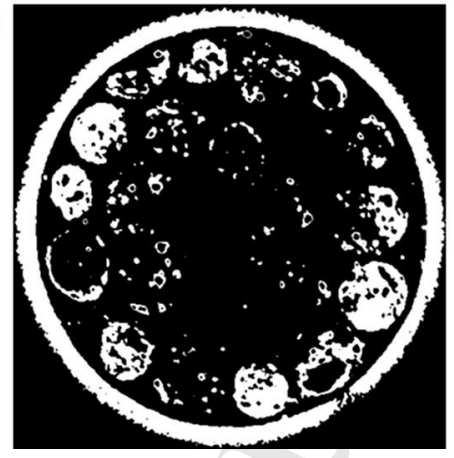

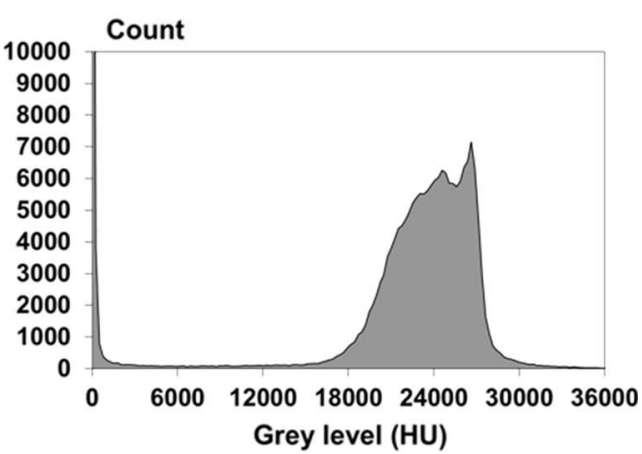
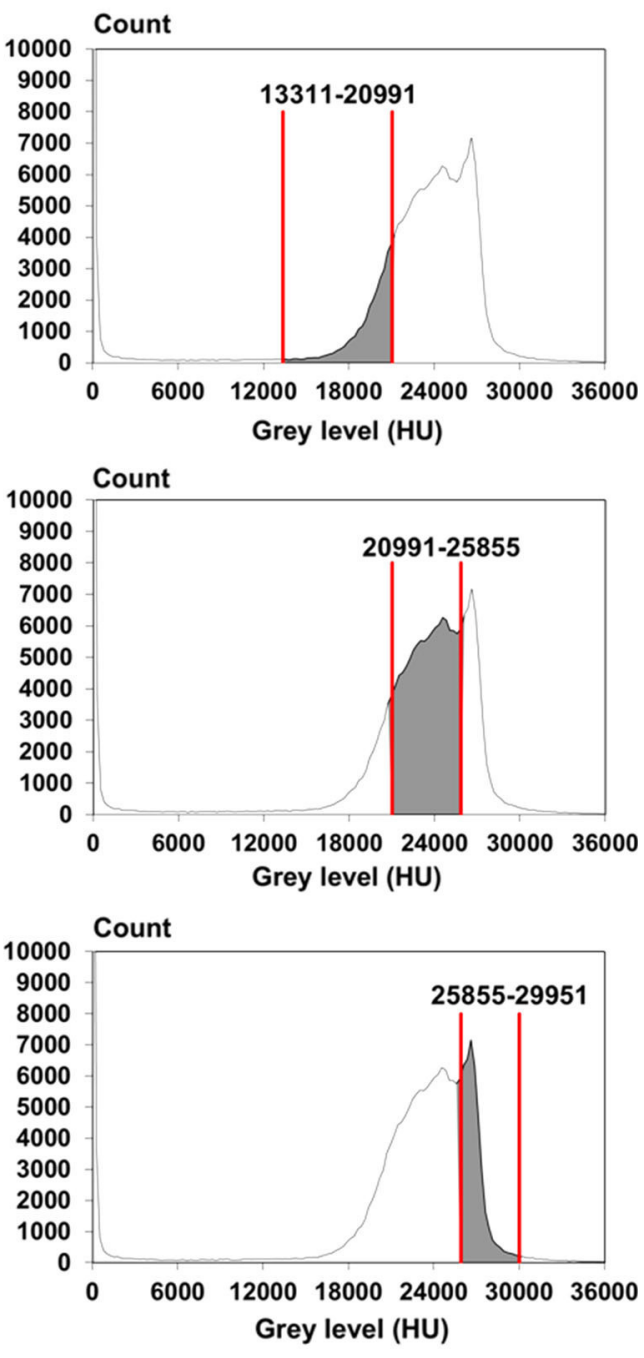

Fig. 5 Image segmentation of the slice no. 1000 at time range 270-295 min of infiltration, with visual images at left and corresponding grey-level band at right for three segmentation ranges: a whole range; b 13,311-20,991; c $20,991-25,855$; d $25,855-29,951$ 
(a)

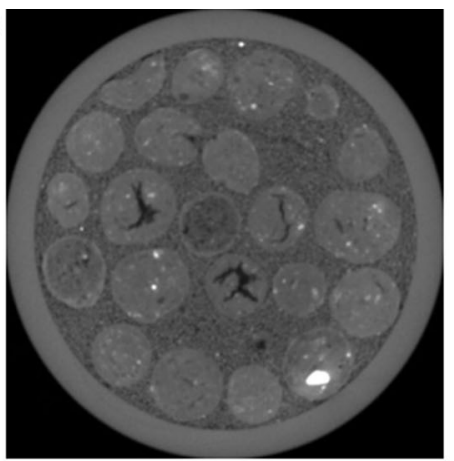

(b)

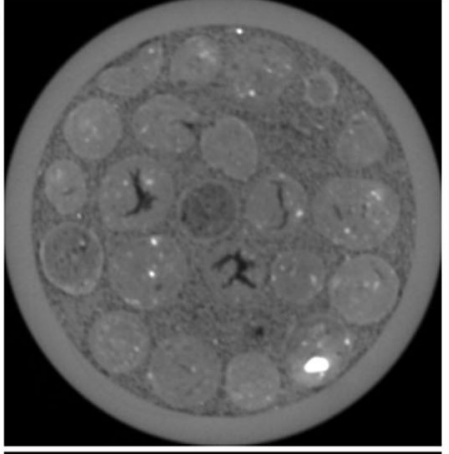

(c)

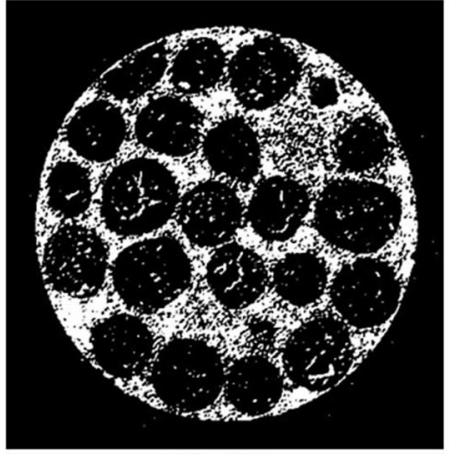

(d)

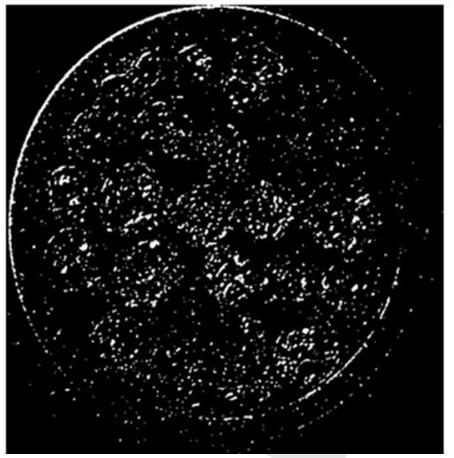

Count
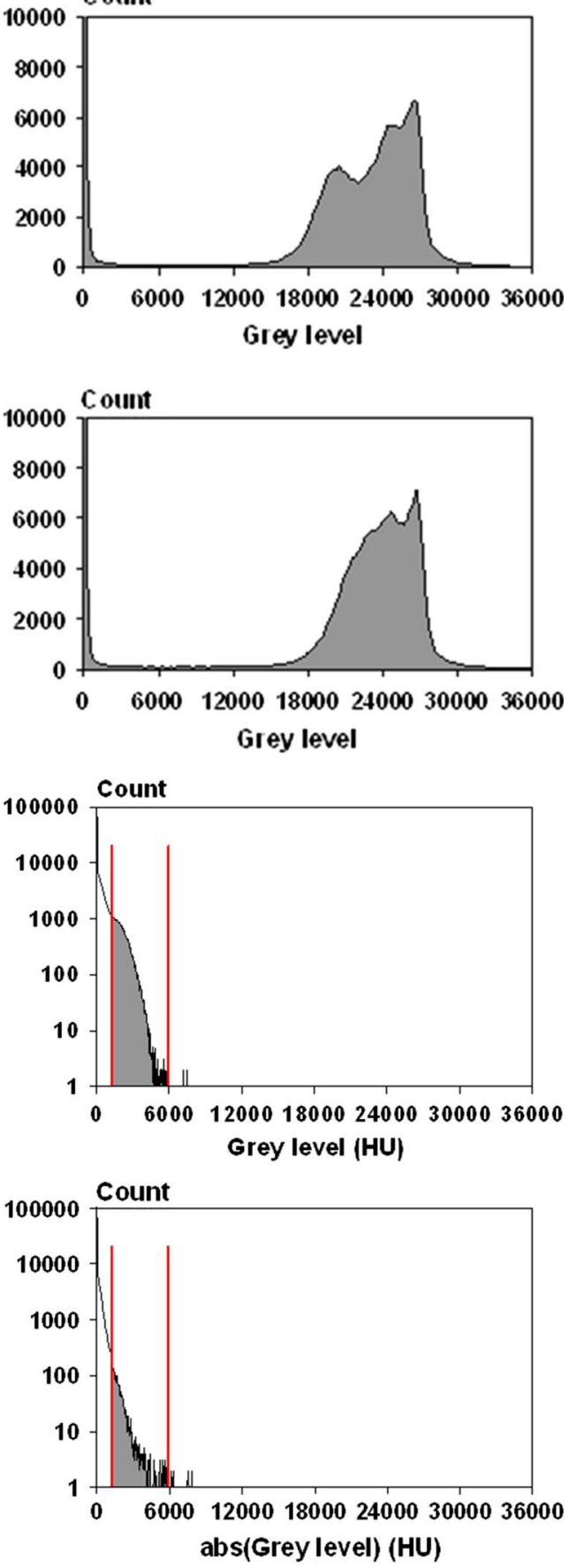

Fig. 6 Example of the slice no. 1000 with visual images at left and corresponding grey level band at right for a slice of dry state; b slice of wet state at time range 270-295 min; Binary subtracted image with c positive grey levels; d negative grey levels 
(a)

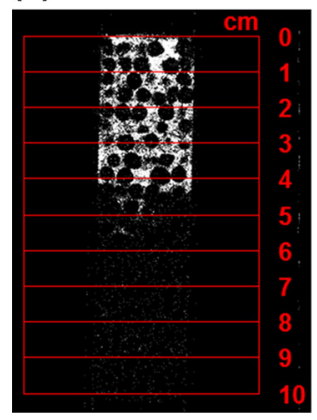

(b)

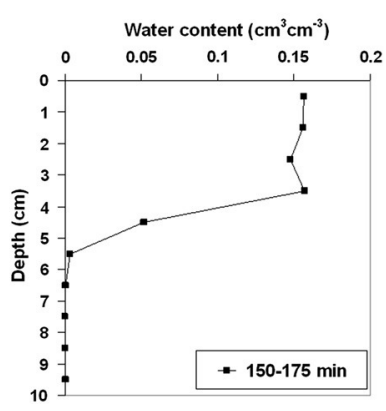

(c)

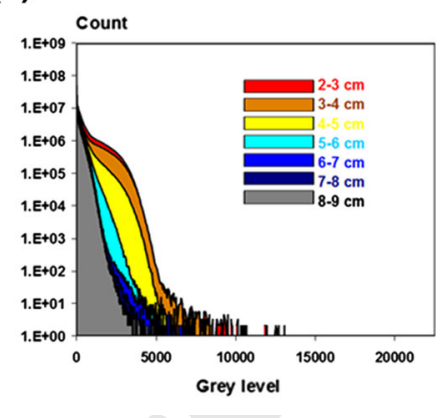

Fig. 7 Example of water content profile obtained with CT images at time range 150-175 min: a vertical CT water profile (subtracted image), the void and filled by water volumes are represented by black and white colours, respectively; b water content profile obtained with 3D CT images: each point showed the average water content per layer of $1 \mathrm{~cm}$ (160 slice per layer); $\mathbf{c}$ the histogram sum of 160 slices comprised in each layer of $1 \mathrm{~cm}$ thickness

opaque mask with low statistic bins count (the range included in red interval, Fig. 6d). We believe that these negative pixels/voxels may relate to the "diffuse matrix" (in Sammartino et al. 2012) that is voxels/pixels which were filled by a combination of air and soil at the dry state and are constituted by a combination of water and soil at time $t$. This combined with microdisplacement of the sand grains below the imaging resolution may result in an apparent voxel grey-level value lower at time $t$, thus creating subtracted image voxels with negative pixel values. This is consistent with our hypothesis in condition (3). As a consequence, the discussion concerning the water transport was rather focused on the image obtained with positive grey levels. Compared to what was obtained by traditional segmentation method, we found that the subtracted image method showed much higher image quality, especially in terms of water distribution.

Finally, all the 1600 slices of subtracted image were segmented and converted in binary format with pixel values 0 and 1 for, respectively, void and water. Considering that all pores are filled with water above the infiltration front, the threshold for binary image segmentation was chosen so that the average water content in wet layers of the last scan (at time range of 270-295 $\mathrm{min}$ ) was close to the theoretical inter-aggregate porosity of the column $3\left(0.181 \mathrm{~cm}^{3} \mathrm{~cm}^{-3}\right)$. Water content of each slice was then calculated as the ratio of water bins count onto the total bins count of column section. An example is shown in Fig. 7: at the time range of 150-175 min during the infiltration, vertical water profile (Fig. 7b) is calculated from water contents averaged over $1 \mathrm{~cm}$ (160 slices) which is about the height of the periodic layer. The subtracted CT image profile (Fig. 7a) is also showed for comparison. The water content profile (Fig. 7b) showed consistent evolution with subtracted CT images (Fig. 7a), i.e. water front lies between 4 and $6 \mathrm{~cm}$ in depth and water content drops to 0 below $6 \mathrm{~cm}$. As well, statistical bins account on the grey-level histograms (Fig. 7c) decreased layer by layer from 0 to $6 \mathrm{~cm}$ (the layers of $0-1$ and $1-2 \mathrm{~cm}$ were not shown because they exhibited the same histogram than the layer of $2-3 \mathrm{~cm}$ ) and reached a minimal account below the layer of $6-7 \mathrm{~cm}$.

\subsection{Evolution of 2D Water Profile and Water Front in 3D}

With lastly mentioned method, evolution of vertical water profiles in column 3 with time is shown in Fig. 8 and compared with corresponding CT images. According to the CT images (Fig. 8 left), sphere voids appeared behind the water front and maintained their void shape 
(a)

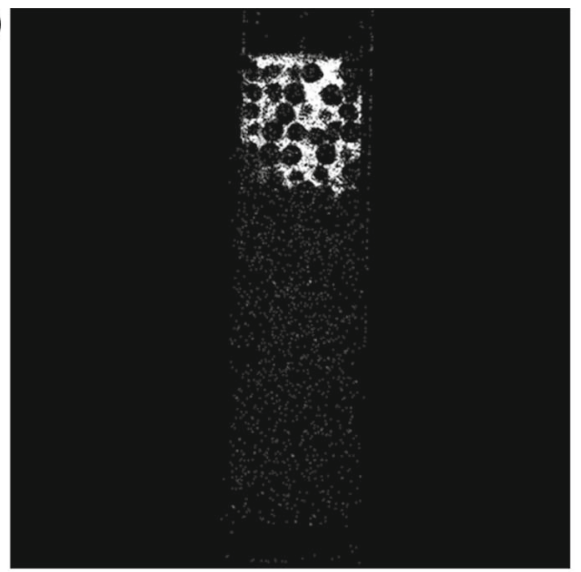

(b)

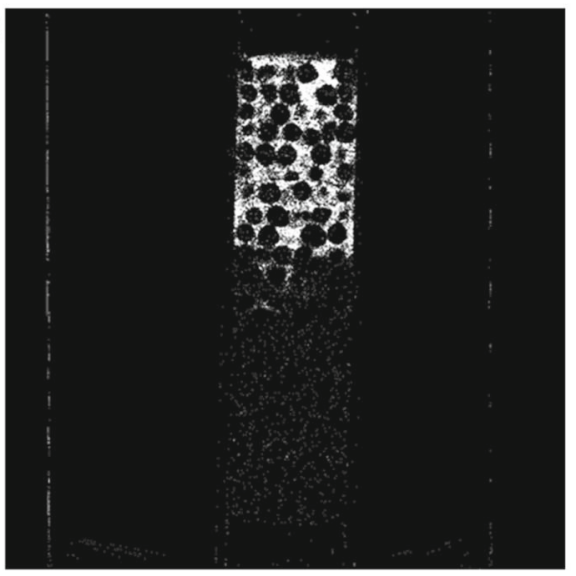

(c)

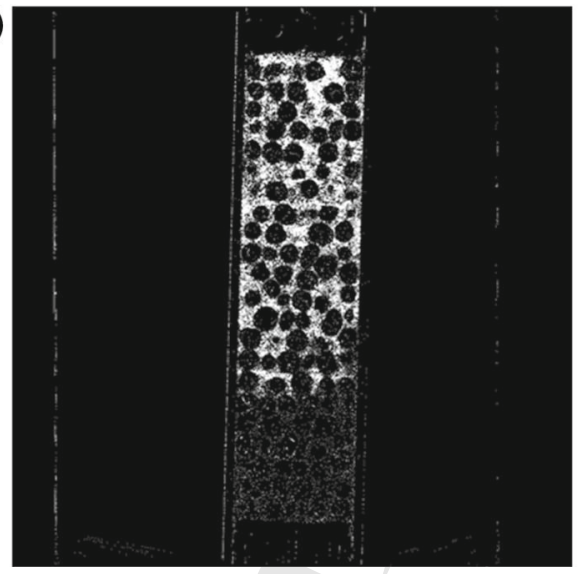

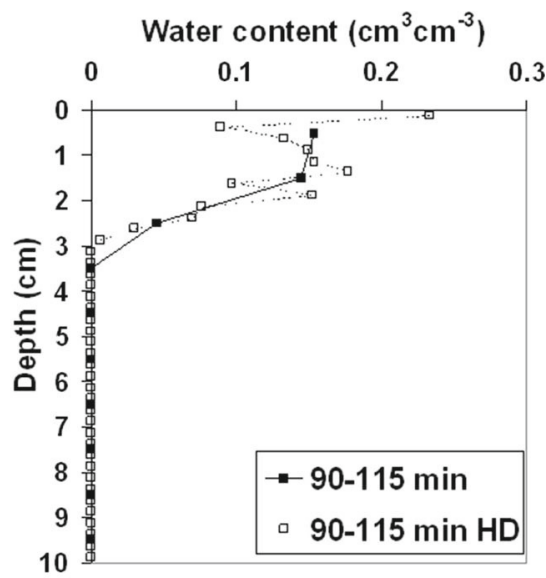

Water content $\left(\mathrm{cm}^{3} \mathrm{~cm}^{-3}\right)$
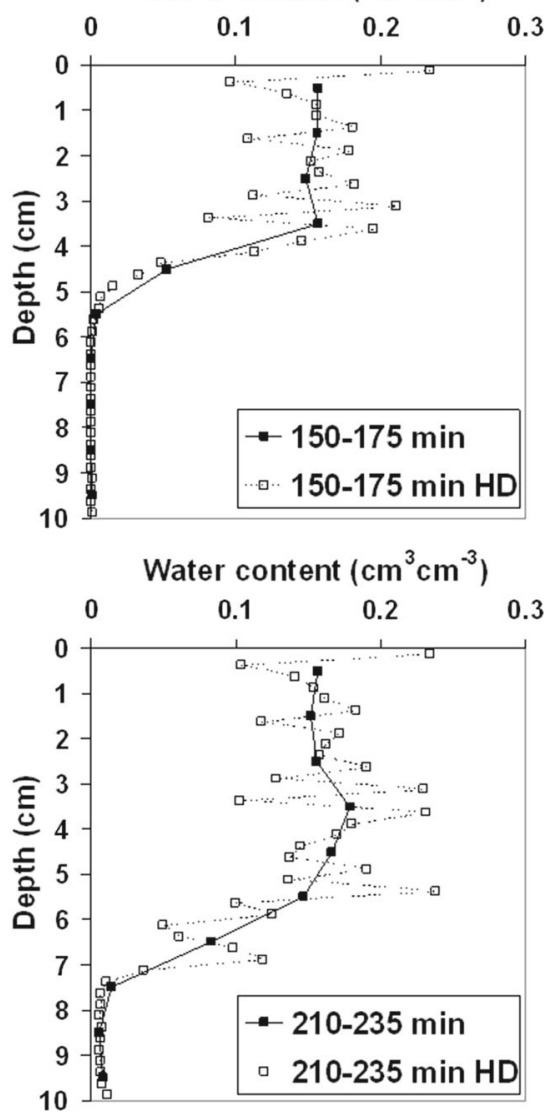

Fig. 8 Left Vertical CT water profiles, the void and filled by water volumes are represented by black and white colours; Right water content profiles, for the time ranges of a 90-115 min, b 150-175 min and c 210-235 $\min$ 


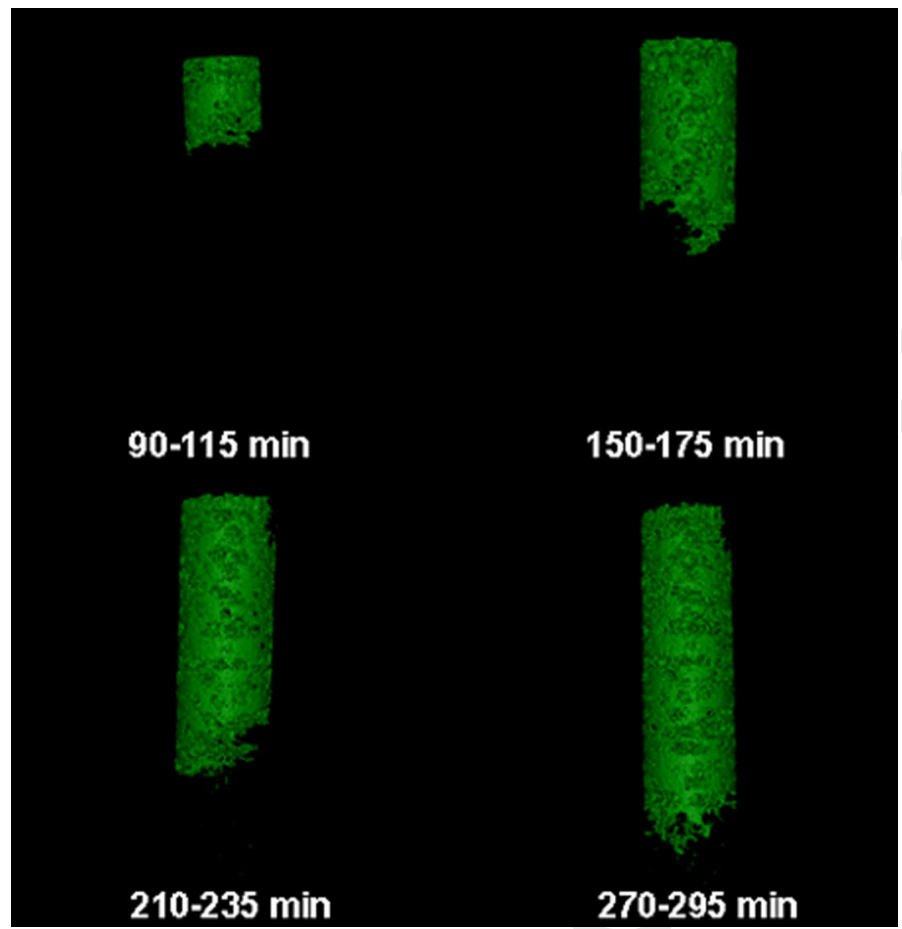

Fig. $93 \mathrm{D}$ water volume reconstruction at different time ranges of the infiltration

along water front advance. These sphere voids correspond logically to clay spheres and showed that little water filled into clay spheres during infiltration. This is consistent with what found in gravimetrical measurement. On the water content profiles (Fig. 8 right), two different control volumes are compared. The low resolution corresponds to the average of water content over around one periodic layer (every $1 \mathrm{~cm}$ ) and the high resolution (HD) to a step of $0.25-\mathrm{cm}$ height. The HD profiles showed clearly fluctuating profiles compared to these of low resolution. This latter can be explained by the sensibility of HD profile associated with local constitution of sand and clay spheres at different layers. As water hardly filled into clay spheres, water content is lower in the layers where more clay spheres are present. As well, while water content is averaged over more slices (low definition), the corresponding water content is found similar to average inter-aggregate porosity of double-porosity media and the profiles exhibit less fluctuation. The periodic layer is thus a good representative elementary volume for water content calculation.

By the 3D reconstruction of subtracted images, we noticed that the water front was heterogeneous (Fig. 9) and is linked to physical non-equilibrium as shown during solute displacement experiment.

\section{Conclusions and Perspectives}

In this study, we developed an efficient method to couple images acquisition by computed tomography technique and water infiltration experiment, in the aim to visualize water distribution in $4 \mathrm{D}$ at submicron scale. In order to overcome the common difficulty in $\mathrm{CT}$ technique, 
i.e. the low temporal resolution vs. to rapid physical phenomena of water transport, we chose carefully the experimental combination among infiltration speed and relatively high temporal and spatial resolution CT configuration.

By first performing macroscopic measurements in a column made of two medium of contrasted hydraulic conductivities (sand and porous clay spheres), we showed that the infiltration of water into the clay spheres was negligible at the observed time scale. The calculation of characteristics times show that water transfer into clay spheres is mainly diffusive at times larger than our infiltration experiment duration.

In order to observe water flow at finer spatial scale, we succeeded to follow the water front displacement by CT imaging, and by a novel image analysis method in soil science, we could extract water distribution in two and three dimensions. The obtained 2D or 3D water distribution showed us that little water filled into clay sphere during the observed time range of infiltration. This is consistent with macroscopic gravimetrical measurements. By averaging the water content over one periodic layer, we could obtain the water content evolution along the column and determine the depth of the water infiltration front, as observed in the subtracted binary images. By reconstructing 3D water distribution, we found that the water front showed a heterogeneous shape which was related to the heterogeneity of the double-porosity media leading to water transfer in physical non-equilibrium.

This work presents an adequate method to study water transport kinetics by CT technique. However, enhancement of experimental set-up is still needed. The spatial resolution of $6 \times 10^{-3} \mathrm{~cm}$ in this work is not high enough to study processes at the pore scale between sand grains and inside clay spheres. The temporal resolution of 25 min compared to 8-h infiltration allowed following the water front well, but still showed an uncertainty on the water front position of 16 voxels or $0.096 \mathrm{~cm}$. To further improve the temporal resolution, we need higher intensity of the radiation (i.e. higher photon particle amount included in a wave packet) while keeping low photon energy for high-transmission-coefficient contrast. This may be realized by grand instrument such as synchrotron facility to image smaller samples (a few millimetres thick). To improve the spatial resolution, a direct remedy is to use a better CDD sensor with higher pixel resolution. Lowering carefully the wavelength of the radiation can provide higher interaction between the radiation and material at small length scale and thus better spatial resolution. However, a decrease in the wavelength leads to increase in the photon energy. This has to be adjusted carefully in order to keep a minimal transmission coefficient contrast.

Acknowledgments This project and post-doc scholarship for Z. Peng have been funded by a specific grant awarded by Grenoble Institute of Technology. We thank J.F. Daian for the help in using XDQ software. This project has also received partial funding from the European Union's Horizon 2020 research and innovation programme under Grant Agreement No. 645717.

\section{References}

Akhtar, M.S., Stüben, D., Norra, S., Memon, M.: Soil structure and flow rate-controlled molybdate, arsenate and chromium(III) transport through field columns. Geoderma 161(3-4), 126-137 (2011)

Akin, S., Schembre, J.M., Bhat, S.K., Kovscek, A.R.: Spontaneous imbibition characteristics of diatomite. J. Pet. Sci. Eng. 25(3-4), 149-165 (2000)

Angulo-Jaramillo, R., Vandervaere, J.P., Roulier, S., Thony, J.L., Gaudet, J.P., Vauclin, M.: Field measurement of soil surface hydraulic properties by disc and ring infiltrometers-a review and recent developments. Soil Till. Res. 55(1-2), 1-29 (2000)

Carminati, A., Kaestner, A., Ippisch, O., Koliji, A., Lehmann, P., Hassanein, R., Vontobel, P., Lehmann, E., Laloui, L., Vulliet, L., Flühler, H.: Water flow between soil aggregates. Transp. Porous Med. 68(2), 219-236 (2007) 
Carminati, A., Kaestner, A., Lehmann, P., Flühler, H.: Unsaturated water flow across soil aggregate contacts. Adv. Water Resour. 31(9), 1221-1232 (2008)

Dal Ferro, N., Delmas, P., Duwig, C., Simonetti, G., Morari, F.: Coupling X-ray microtomography and mercury intrusion porosimetry to quantify aggregate structures of a cambisol under different fertilisation treatments. Soil Till. Res. 119, 13-21 (2012)

Desaunay, A.: Etude et modélisation de la biosorption des métaux par les bactéries. Application au transfert du cadmium et du zinc, seuls ou en mélange, par Escherichia coli et Cupriavidus metallidurans en colonnes de sable d'Hostun. PhD of Joseph Fourier university (2011)

DiCarlo, D.A., Seale, L.D., Ham, K., Willson, C.S.: Tomographic measurements of pore filling at infiltration fronts. Adv. Water Resour. 33(4), 485-492 (2010)

Elyeznasni, N., Sellami, F., Pot, V., Benoit, P., Vieublé-Gonod, L., Young, I., Peth, S.: Exploration of soil micromorphology to identify coarse-sized OM assemblages in X-ray CT images of undisturbed cultivated soil cores. Geoderma 179, 38-45 (2012)

Esser, H.G., Carminati, A., Vontobel, P., Lehmann, E.H., Oswald, S.E.: Neutron radiography and tomography of water distribution in the root zone. J. Plant Nutr. Soil Sci. 173(5), 757-764 (2010)

Heindel, T.J., Gray, J.N., Jensen, T.C.: An X-ray system for visualizing fluid flows. Flow Meas. Instrum. 19(2), 67-78 (2008)

Herman, G.T.: Fundamentals of Computerized Tomography: Image Reconstruction from Projections, 2nd edn. Springer Publishing Company Inc., New York (2009)

Holtham, D.A.L., Matthews, G.P., Scholefield, D.S.: Measurement and simulation of void structure and hydraulic changes caused by root-induced soil structuring under white clover compared to ryegrass. Geoderma 142(1-2), 142-151 (2007)

Hopmans, J.W., Vogel, T., Koblik, P.D.: X-ray tomography of soil-water distribution in one-step outflow experiments. Soil Sci. Soc. Am. J. 56(2), 355-362 (1992)

Kneafsey, T.J., Seol, Y., Gupta, A., Tomutsa, L.: Permeability of laboratory-formed methane-hydrate-bearing sand: measurements and observations using X-ray computed tomography. SPE J. 16(1), 78-94 (2011)

Lewandowska, J., Szymkiewicz, A., Burzynski, K., Vauclin, M.: Modeling of unsaturated water flow in doubleporosity soils by the homogenization approach. Adv. Water Resour. 27(3), 283-296 (2004)

Lewandowska, J., Szymkiewicz, A., Auriault, J.: Upscaling of Richards' equation for soils containing highly conductive inclusions. Adv. Water Resour. 28(11), 1159-1170 (2005)

Lewandowska, J., Szymkiewicz, A., Gorczewska, W., Vauclin, M.: Infiltration in a double-porosity medium: experiments and comparison with a theoretical model. Water Resour. Res. 41(2), W02022 (2005bis)

Lewandowska, J., Tran Ngoc, T.D., Vauclin, M., Bertin, H.: Water drainage in double-porosity soils: experiments and micro-macro modeling. J. Geotech. Geoenviron. 134(2), 231-243 (2008)

Lugato, E., Morari, F., Nardi, S., Berti, A., Giardini, L.: Relationship between aggregate pore size distribution and organic-humic carbon in contrasting soils. Soil Till. Res. 103(1), 153-157 (2009)

Luo, L., Lin, H., Li, S.: Quantification of 3-D soil macropore networks in different soil types and land uses using computed tomography. J. Hydrol. 393, 53-64 (2010)

Markowicz, A.A.: X-ray physics. In: Van Grieken, R.E., Markowicz, A.A. (eds.) Handbook of X-Ray Spectrometry, pp. 1-28. Marcel Dekker, New York (1993)

Maruyama, T., Tada, A., Iwama, K., Horino, H.: Direct observation of soil water movement through soil macropores using soft X-rays and stereographing. Soil Sci. 168(2), 119-127 (2003)

Munkholm, L.J., Heck, R.J., Deen, B.: Soil pore characteristics assessed from X-ray micro-Cf derived images and correlations to soil friability. Geoderma 181, 22-29 (2012)

Sammartino, S., Michel, E., Capowiez, Y.: A novel method to visualize and characterize preferential flow in undisturbed soil cores by using multislice helical CT. Vadose Zone J. (2012). doi:10.2136/vzj2011.0100

Sato, T., Tanahashi, H., Loaiciga, H.A.: Solute dispersion in a variably saturated sand. Water Resour. Res. 39(6), 1155-1162 (2003)

Schjonning, P., Iversen, B.V., Munkholm, L.J., Labouriau, R., Jacobsen, O.H.: Pore characteristics and hydraulic properties of a sandy loam supplied for a century with either animal manure or mineral fertilizers. Soil Use Manage. 21(3), 265-275 (2005)

Schaap, J.D., Lehmann, P., Kaestner, A., Vontobel, P., Hassanein, R., Frei, G., de Rooij, G.H., Lehmann, E., Flühler, H.: Measuring the effect of structural connectivity on the water dynamics in heterogeneous porous media using speedy neutron tomography. Adv. Water Resour. 31(9), 1233-1241 (2008)

Schneider, C.A., Rasband, W.S., Eliceiri, K.W.: NIH Image to ImageJ: 25 years of image analysis. Nat. Methods 9(7), 671-675 (2012)

Serra, J.: Image Analysis and Mathematical Morphology. Academic Press, Inc., Orlando (1983)

Sleutel, S., Cnudde, V., Masschaele, B., Vlassenbroek, J., Dierick, M., Van Hoorebeke, L., Jacobs, P., De Neve, S.: Comparison of different nano- and micro-focus X-ray computed tomography set-ups for the visualization of the soil microstructure and soil organic matter. Comput. Geosci. 34(8), 931-938 (2008) 
Szymkiewicz, A., Lewandowska, J., Angulo-Jaramillo, R., Butlanska, J.: Two-scale modeling of unsaturated water flow in a double-porosity medium under axisymmetric conditions. Can. Geotech. J. 45(2), 238-251 (2008)

Szymkiewicz, A., Lewandowska, J.: Micromechanical approach to unsaturated water flow in structured geomaterials by two-scale computations. Acta Geotech. 3(1), 37-47 (2008)

Toride, N., Leij, F.J., van Genuchten, M.Th.: The CXTFIT Code for Estimating Transport Parameters from Laboratory or Field Tracer Experiments. Version 2.1. U.S. Salinity Laboratory, Riverside, California (1999)

Toride, N., Inoue, M., Leij, F.J.: Hydrodynamic dispersion in an unsaturated dune sand. Soil Sci. Soc. Am. J. 67, 703-712 (2003)

Tran Ngoc, T.D.: Transport de solutés dans un milieu à double-porosité non saturé. Modélisation par homogénéisation \& applications. PhD of Joseph Fourier university (2008)

Vasin, M., Lehmann, P., Kaestner, A., Hassanein, R., Nowak, W., Helmig, R., Neuweiler, I.: Drainage in heterogeneous sand columns with different geometric structures. Adv. Water Resour. 31(9), 1205-1220 (2008)

Vogel, H.J., Roth, K.: Moving through scales and flow and transport in soil. J. Hydrol. 272(1-4), 95-106 (2003)

Wildenschild, D., Hopmans, J.W., Rivers, M.L., Kent, A.J.R.: Quantitative analysis of flow processes in a sand using synchrotron-based X-ray microtomography. Vadose Zone J. 4(1), 112-126 (2005)

Wildenschild, D., Sheppard, A.P.: X-ray imaging and analysis techniques for quantifying pore-scale structure and processes in subsurface porous medium systems. Adv. Water Resour. 51, 217-246 (2013)

Withjack, E.M.: Computed tomography for rock-property determination and fluid-flow visualization. Soc. Pet. Eng. Form. Eval. 3, 696-704 (1988)

$\mathrm{Xu}, \mathrm{K}$., Daian, J.F., Quenard, D.: Multiscale structures to describe porous media. Part II: transport properties and application to test materials. Transp. Porous Med. 26(3), 319-338 (1997) 


\section{Author Query Form}

\section{Please ensure you fill out your response to the queries raised below and return this form along with your corrections}

\section{Dear Author}

During the process of typesetting your article, the following queries have arisen. Please check your typeset proof carefully against the queries listed below and mark the necessary changes either directly on the proof/online grid or in the 'Author's response' area provided below

\begin{tabular}{|c|l|l|}
\hline Query & \multicolumn{1}{|c|}{ Details required } & Author's response \\
\hline 1. & $\begin{array}{l}\text { Please check the term 'X-Rays Com- } \\
\text { puted Tomography' that has been } \\
\text { changed to 'X-ray Computed Tomogra- } \\
\text { phy' in the article title. }\end{array}$ & \\
\hline 2. & $\begin{array}{l}\text { Please provide significance for super- } \\
\text { script b in Table 2. }\end{array}$ & \\
\hline 3. & $\begin{array}{l}\text { Reference Serra (1982) has been } \\
\text { changed to Serra (1983), so that this } \\
\text { citation matches the list. }\end{array}$ & \\
\hline 4. & $\begin{array}{l}\text { Please provide the year for the phrase } \\
\text { 'late 80's' in the sentence 'This method is } \\
\text { used currently in petroleum engineering } \\
\text { since the late 80's'. }\end{array}$ & \\
\hline 5. & $\begin{array}{l}\text { Please check and confirm that unit "cm3 } \\
\text { cm-3" repeated in text is correct. }\end{array}$ & \\
\hline
\end{tabular}

